\title{
Hoplitis lithodorae MÜLLER 2012 (Apoidea - Megachilidae - Osmiini) en France
}

\author{
par Matthieu AUBERT*
}

\begin{abstract}
Résumé. L'auteur relate ici la mise en évidence au sein de la frange méditerranéenne de France métropolitaine d'une espèce d'Osmiini décrite récemment d'Espagne : Hoplitis lithodorae MÜLLER 2012. Ses différentes stations françaises connues sont indiquées. Sa biologie, son écologie puis sa distribution sont ensuite brièvement présentées. Quelques éléments pour sa détermination sont finalement consignés dans la perspective de mieux connaître ce taxon, et plus largement le fascinant groupe d'abeilles auquel il appartient.
\end{abstract}

Abstract. Hoplitis lithodorae MÜLLER 2012, an osmiine bee species recently described from Spain, has been discovered in southern France. Its known localities in France are listed here, as are briefly discussed its distribution, biology and ecology. In order to improve our knowledge of this species and about its fascinating genus, some indications to perform identifications are finally presented.

Mots-clés : Hoplitis ; France ; Lithodora ; distribution.

\section{Introduction}

Le genre Hoplitis KLUG appartient à la tribu des Osmiini qui correspond aux «Osmies » au sens le plus large. Elle forme avec celles des Megachilini et des Anthidiini la plus grande part de la famille des Megachilidae (MichENER, 2007). Cette dernière est caractérisée notamment par la présence chez les femelles de la brosse de transport du pollen sous l'abdomen, du moins chez les taxons non parasites. Certains auteurs ont conservé les Hoplitis au sein du genre Osmia PANZER, au même titre que d'autres taxons proches. C'est le cas par exemple dans les clés proposées par BENOIST (1931) ou plus récemment par AMIET et al. (2004). Toutefois, MiCHENER (2007) et MÜLLER (2013) considèrent les Hoplitis comme un genre à part entière.

Le genre compte plus de 350 espèces dans le Monde et se trouve très diversifié au sein de l'aire paléarctique occidentale : on y en dénombre plus de 200 (KUHLMANN et al., 2014). Il est divisé en de nombreux sous-genres, les plus importants

* Matthieu AUBERT

Route de Pégairolles, 34380 SAINT-JEAN-DE-BUEGES

Contact : matthieu@oabeilles.net numériquement en Europe étant les sous-genres Alcidamea CREsson, Anthocopa LEPELETIER \& SERVILLE et Hoplitis KLUG s. str. ; ceux-ci étant représentés en France par une dizaine d'espèces chacun. D'autres sous-genres, moins riches, s'y trouvent également (Formicapis SLADEN et Micreriades MAVROMOUSTAKIS ; voir SEDIVY et al., 2013 pour plus de détails). Au total, 36 espèces du genre Hoplitis ont été recensées jusqu'à présent en France, Corse comprise (RASMONT et al., 1995 ; MÜLLER, 2013 ; LE GOFF comm. pers.).

\section{Hoplitis (Hoplitis) lithodorae MÜLLER en France}

Plusieurs taxons appartenant à ce groupe ont été décrits récemment d'Espagne (MÜLLER, 2012), parmi lesquels Hoplitis (Hoplitis) lithodorae MÜLLER. Des chasses à vue menées au printemps 2013 dans les environs de Montpellier (34) sur le chaînon de la Gardiole et sur les contreforts du Causse d'Aumelas y ont révélé l'espèce :

01.V.13 - Fabrègues (34), Pioch de Camille $43.51^{\circ} \mathrm{N}, 3.75^{\circ} \mathrm{E}, 130 \mathrm{~m}$ - réc. : C. Philippe \& $\mathrm{M}$. Aubert - dét. : A. Müller : 1 m. et 1 f. (coll. G. Le Goff), 1 m. (coll. M. Aubert), 1 m. (coll. C. Philippe). 


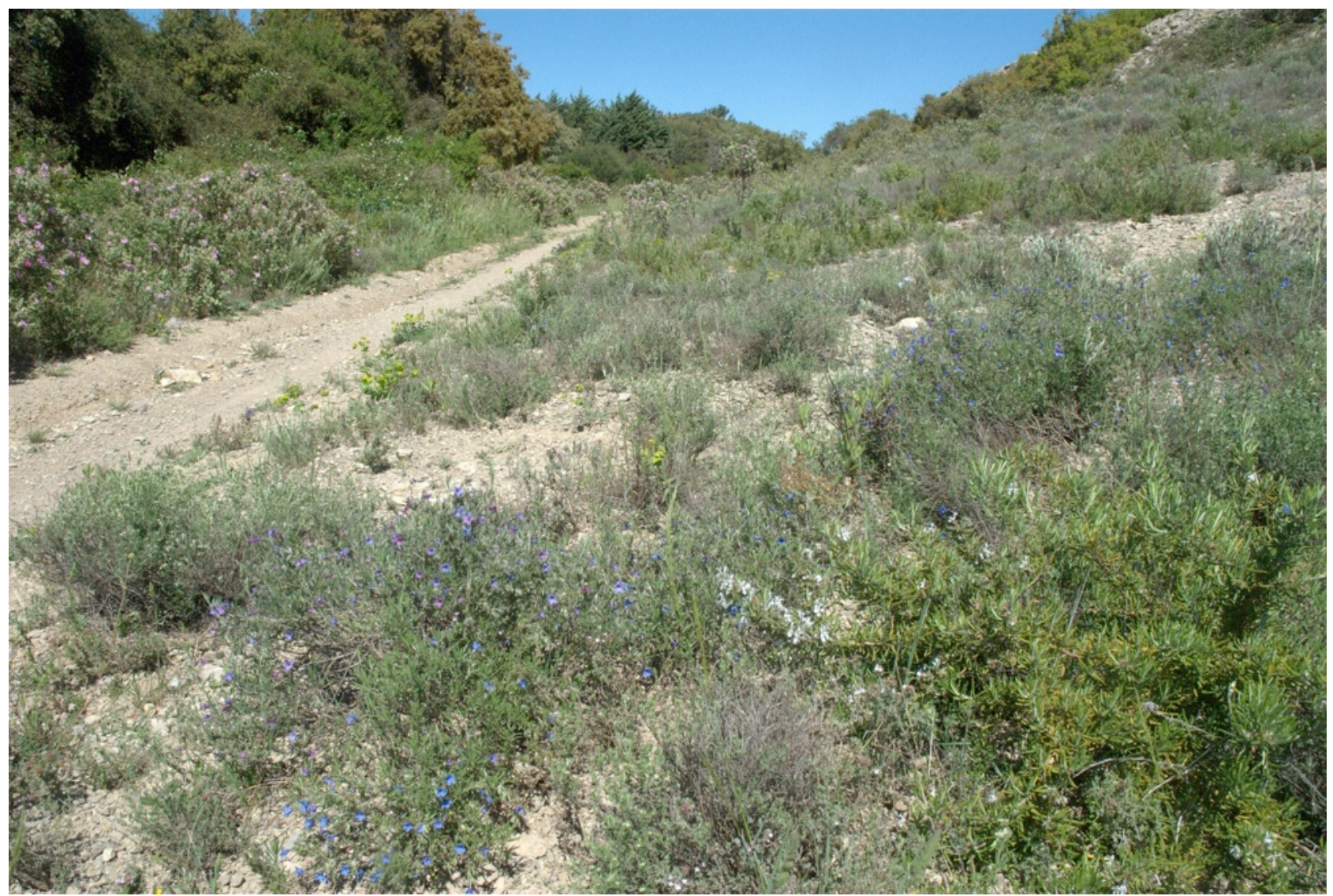

Figure 1. Habitat d'Hoplitis lithodorae (Hym. Megachilidae). Photo M. Aubert.

02.V.13 - Aumelas (34), Cabrials - $43.55^{\circ} \mathrm{N}$, $3.57^{\circ} \mathrm{E}, 100 \mathrm{~m}$ - réc. : C. Philippe \& M. Aubert dét. : A. Müller : 1 f. (coll. M. Aubert), 1 m. (coll. C. Philippe).

Les quelques connaissances acquises jusqu'alors concernant son écologie ont permis d'orienter sa recherche les deux années suivantes. C'est ainsi qu'H. lithodorae a été mise en évidence également dans l'Aude et les Bouches-du-Rhône au printemps 2014 et que plusieurs autres stations ont été découvertes dans le nord-est de l'Hérault en 2015 :

16.IV.14 - Montbrun-des-Corbières (11) $43.19^{\circ} \mathrm{N}, 2.68^{\circ} \mathrm{E}, 150 \mathrm{~m}$ - réc. : D. Genoud - dét. : D. Genoud : 1 m. (coll. D. Genoud).

15.IV.14 - Cabriès (13) - $43.47^{\circ} \mathrm{N}, 5.34^{\circ} \mathrm{E}, 170 \mathrm{~m}$ réc. : M. Aubert - dét. : M. Aubert : 1 m. (coll. M. Aubert).

08.IV.15 - Ferrières-les-Verreries (34), proximité de l'ancienne verrerie $-44.83^{\circ} \mathrm{N}, 3.80^{\circ} \mathrm{E}, 300 \mathrm{~m}$ réc. : E. Dufrêne - dét. : M. Aubert : 2 m. (coll. E. Dufrêne).

15.IV.15 - Saint-Jean-de-Buèges (34), Les Graves - $43,84^{\circ} \mathrm{N}, 3.62^{\circ} \mathrm{E}, 270 \mathrm{~m}$ - réc. : M. Aubert - dét. : M. Aubert : 1 m. (coll. M. Aubert).

\section{Éléments de biologie et d'écologie}

Un grand nombre d'Hoplitis sont spécialisées sur un groupe restreint de plantes pour la récolte du pollen. C'est le cas par exemple d'Hoplitis (Hoplitis) adunca (PANZER 1798), l'espèce du genre la plus largement répandue et la plus fréquente dans notre pays. Les femelles prélèvent en effet le pollen nécessaire à leur progéniture exclusivement sur des vipérines (Echium L. spp.). MÜLLER (2012, 2013) suggère qu'H. lithodorae est elle-même très probablement oligolectique sur les Grémils (Lithodora Griseb spp.), qui sont également des Boraginaceae. Nos observations vont dans le sens de cette hypothèse : tous les spécimens considérés ont été observés et attrapés systématiquement au niveau de stations de Lithodora fruticosa (L.), à la période de sa floraison. C'est précisément ce qui a dirigé nos recherches. L'analyse d'un nombre plus important de charges polliniques portées par des femelles, et provenant d'un nombre plus grand de stations, permettra plus rigoureusement de confirmer ou non une spécialisation. Mais comme nous le verrons plus avant, une caractéristique particulière de l'anatomie des femelles laisse peu de doute sur la question. Il est raisonnable ainsi d'affirmer 
qu'H. lithodorae présente une nette préférence pour les Lithodora. Sur cette base et vu les lieux de récoltes, on peut aussi affirmer qu'elle fréquente, sinon exclusivement au moins préférentiellement, des habitats ouverts chauds et secs sur substrat calcaire (typiquement des pelouses et garrigues basses rocailleuses). Une des stations de l'espèce est illustrée sur la figure 1. Sa nidification n’a pas encore été observée.

\section{Distribution}

La description d'H. lithodorae a été réalisée sur la base de spécimens provenant de la marge méditerranéenne de l'Espagne : sud de Valence, région autonome de Murcia et extrême est de l'Andalousie (MÜLLER, 2012). Sa distribution telle qu'elle nous est connue aujourd'hui comprend ce secteur espagnol et un second qui s'étend de la marge orientale des Corbières aux environs d'Aix-en-Provence. Cependant, $H$. lithodorae est très certainement plus largement répartie, tout au moins dans le sud de l'Espagne et de la France. Elle est donc à rechercher bien entendu entre les deux zones géographiques citées précédemment mais aussi au-delà, à l'ouest de la région limitrophe entre les provinces autonomes de Murcia et d'Andalousie et à l'est de l'Etang de Berre au moins jusque dans le Var où se trouve encore assez fréquemment $L$. fruticosa. Par ailleurs, compte tenu de sa description récente, rajoutons qu'il n'est pas impossible que des exemplaires de cette espèce se trouvent dans

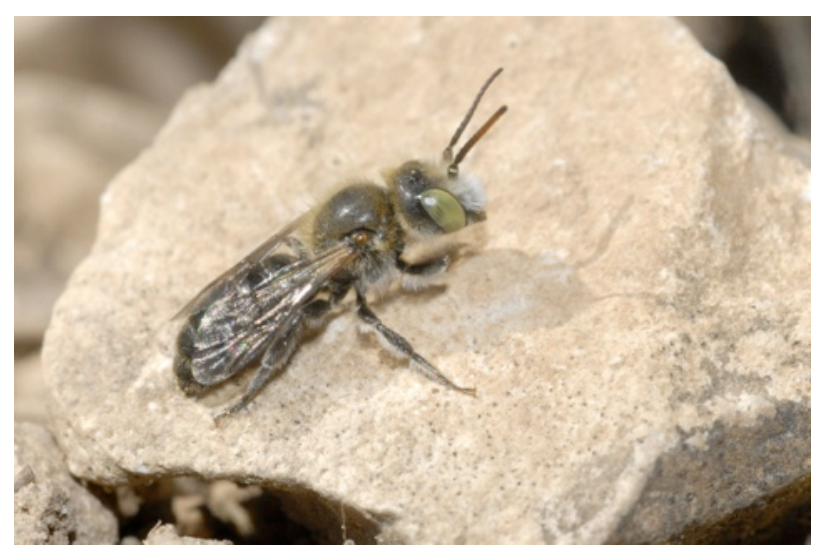

Figure 2. Mâle d'Hoplitis lithodorae. Photo M. Aubert.

des collections existantes, restés indéterminés ou pris pour une autre. Ainsi, en complément des recherches de terrain, certaines données se trouvent potentiellement déjà chez vous si vous avez récolté des Hoplitis en zone méditerranéenne occidentale.

\section{Éléments de détermination}

H. lithodorae est de taille moyenne : 8 à $9 \mathrm{~mm}$ pour les femelles et 7 à $9 \mathrm{~mm}$ pour les mâles. Les premières ressemblent à celles d'Hoplitis (Hoplitis) anthocopoides SCHENCK 1853 ou Hoplitis (Hoplitis) loti MoRAWITZ 1867 mais sont munies sur la galéa de petits poils crochus à leur extrémité. Ce caractère, unique pour la faune de France dans ce genre, est sans grand doute en lien avec la spécialisation florale de l'espèce. Il servirait à récupérer le pollen à l'intérieur de la corolle des Grémils. La détermination des mâles peut être assurée quant à elle en examinant la

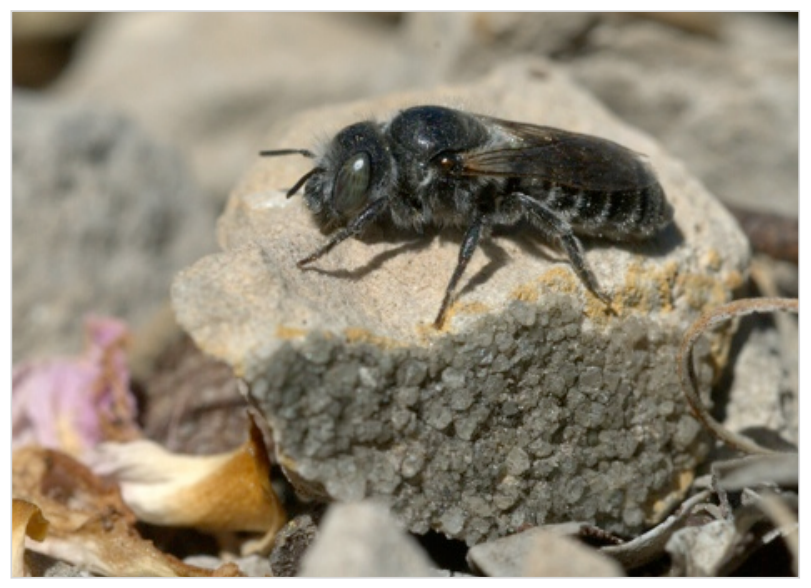

Figure 3. Femelle d'Hoplitis lithodorae. Photo M. Aubert.

marge membraneuse du sternite 6 , composée de deux lamelles trapézoïdales. Pour plus de détails, j'invite le lecteur à se reporter à la publication de MÜLLER (2012) qui comporte la diagnose et des photos et, selon son expérience, à faire vérifier ses identifications.

\section{Remerciements}

Sans l'aide en particulier d'Andreas Müller et de Gérard Le Goff, cet article ne serait sans doute pas ou son auteur serait un autre. Le premier a bien voulu déterminer, entre autres, les premiers spécimens considérés ici, qui m'ont donné bien $\mathrm{du}$ fil à retordre. Le second me permet depuis plusieurs années d'avancer dans l'étude des Megachilidae. Je les en remercie très chaleureusement. Un grand merci aussi à Christophe Philippe et Pascal Auda, compagnons de terrain à la motivation sans faille, de même qu'à David Genoud et Eric Dufrêne pour la transmission de leurs données. 


\section{Références bibliographiques}

Amiet F, Herrmann M, Müller A \& NeUmeYer R, 2004. Fauna Helvetica. Apidae 4. Anthidium, Chelostoma, Coelioxys, Dioxys, Heriades, Lithurgus, Megachile, Osmia, Stelis. Centre Suisse de Cartographie de la Faune, Neuchâtel.

BEnoist R, 1931. Les osmies de la faune française (Hymenopt. Apidae). Annales de la Société Entomologique de France 100, 23-60.

Kunlmann M, et al. Checklist of the Western Palaearctic Bees (Hymenoptera: Apoidea: Anthophila). http://westpalbees.myspecies.info (consulté le 01/01/2016).

Michener CD, 2007. The Bees of the World. $2^{\text {nd }}$ ed. John Hopkins University Press, Baltimore.

Müller A, 2012. New European bee species of the tribe Osmiini (Hymenoptera: Apoidea: Megachilidae). Zootaxa 3355: 29-50.

Müller A, 2013. Palaearctic osmiine bees. Systematics and biology of a fascinating group of solitary bees. ETH Zürich. Disponible à l'adresse http://blogs.ethz.ch/osmiini/ (consulté le 01/01/2016).

RASMont P, Ebmer AW, BANAZsak J, VAN DER ZANDEN G, 1995. Hymenoptera Apoidea Gallica. Liste taxonomique des abeilles de France, de Belgique, de Suisse et du GrandDuché de Luxembourg. Bulletin de la Société Entomologique de France (hors série) 100 : 1-98.

Sedivy C, DoRn S, MÜLler A, 2013. Molecular phylogeny of the bee genus Hoplitis (Megachilidae : Osmiini) - how does nesting biology affect biogeography? Zoological Journal of the Linnean Society 167, 28-42.

Tison JM, JAuZein P, Michaud H, 2014. Flore de la France méditerranéenne continentale. Naturalia publications, Turriers.

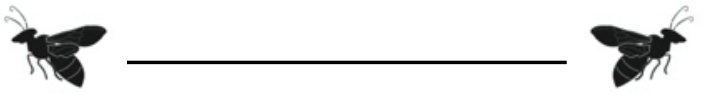

